\title{
Efecto de las exodoncias en el perfil blando de pacientes tratados ortodóncicamente con y sin extracciones
}

\author{
Soft profile changes in patients treated with and without extractions
}

Herbert Orrego Carrillo ${ }^{1, a, b, c}$

\section{RESUMEN}

Objetivos: Se realizó un estudio retrospectivo comparativo de 24 pacientes tratados en una clínica privada con y sin extracciones. Se estudiaron 18 indicadores, a fin de evaluar los cambios que experimentaron a nivel del perfil blando. Material y métodos: La evaluación fue realizada en radiografías cefalométricas pre y post operatorias de ambos grupos. Se emplearon tres líneas referenciales para la evaluación ántero posterior: la línea LVR, la línea B y la línea $\mathrm{E}$ (posterior, media y anterior, respectivamente). La evaluación vertical se realizó analizando la distancia Sn-Me. La prueba estadística empleada fue t de Student. Resultados: En el análisis dentro de cada grupo de estudio se observó que en el grupo tratado sin extracciones los cambios se verificaron fundamentalmente a nivel del labio superior, mostrando una protrusión con respecto a la referencia posterior y a la línea E. Verticalmente, hubo un aumento en la magnitud de la distancia Sn-Me. En el grupo tratado con extracciones, se observó que las variaciones mostraron un aumento de los valores correspondientes a la referencia posterior por parte de ambos labios, mostrando una protrusión de ambos, pero exhibiendo una retrusión de los mismos al ser analizados contra las referencias media (línea B) y anterior (línea A). Verticalmente, se apreció también un cambio en el tercio inferior de la cara, que mostró un aumento de tamaño. Conclusiones: No existieron cambios estadísticamente significativos al comparar los perfiles de pacientes tratados ortodónticamente con y sin extracciones.

PALABRAS CLAVE: Perfil facial, tratamiento ortodóntico sin extracciones, tratamiento ortodóntico con extracciones, cefalometría. (DeCS, BIREME)

Facultad de Odontología, Universidad Nacional Mayor de San Marcos. Lima, Perú.

Diplomado en Ortodoncia.

Especialista en Ortodoncia y Ortopedia Maxilar.

Magister en Estomatología. 


\section{SUMMARY}

Objective: An retrospective comparative study was made in treated patients with and without tooth extractions during their orthodontic therapy, analyzing 18 indicators so it would be possible to evaluate which soft tissue profile changes they experimented. Methods: They were used three reference lines for posterior anterior evaluation: LVR line, B line and E line (posterior, medial and anterior lines respectively). Vertical evaluation was made through Sn-Me distance. Evaluation was made using pre and postoperative cephalometric radiographies from both groups. We used the Student's t-test for the stadistic evaluation. Results: Analyzing each group, we found that most changes were in the upper lip in the non-tooth extractions group showing a protrusion with regard to posterior reference and $E$ line. Vertically, there was an increase in Sn-Me distance. In the tooth extractions group we found that there was an increase in both lips with regard to posterior reference, showing protrusion both of them. However, there was a retrusion compared to medial reference (B line) and anterior reference (A line). Vertically, there was a change in facial lower third which showed an increase of height. Conclusions: There were not any statistically significant changes comparing tooth extractions treated patients with non-tooth extractions treated patients.

\section{KEYWORDS: Facial profile, orthodontic non extraction therapy, orthodontic treatment with extractions, cephalometric. (MeSH, NLM)}

\section{INTRODUCCIÓN}

Las extracciones de primeras premolares son largamentelas más empleadas en la clínica ortodóncica; esta medida terapéutica busca proporcionar las facilidades para solucionar el problema oclusal que presenta un paciente en particular. Sin embargo, aisladamente no garantiza la obtención de un tratamiento exitoso, pues podemos obviar problemas funcionales y estéticos si no hacemos un adecuado diagnóstico del problema maloclusivo integral (1).

Aquella premisa, que si eran adecuadamente colocadas la totalidad de las piezas dentarias, la función y la estética serían conseguidas simultáneamente, ha quedado desestimada. La práctica clínica nos presenta una nueva tarea: analizar a nuestros pacientes desde el punto de vista de los tejidos duros, de los tejidos blandos y de los aspectos funcionales del sistema estomatognático; y a partir de este análisis, plantear las mejores alternativas terapéuticas (2).

Los tratamientos en los que se involucran extracciones han sido señalados por algunos autores $(3,4)$ como provocadores de perfiles poco favorables; otros $(2,5,6,7)$ señalan, que las extracciones terapéuticas no tienen influencia nociva en los perfiles obtenidos luego de la terapia.

El análisis del perfil facial de un paciente antes y después de un tratamiento ortodóncico, nos debe llevar a algunas reflexiones: a) Primero, que los pacientes mayoritariamente procuran tratamiento para mejorar su estética dental; será entonces obligación del profesional informarles que prioritariamente se buscará restituir una adecuada función del sistema estomatológico. b) En segundo lugar, actualmente sabemos con seguridad que el tratamiento influye en la parte facial del paciente; es nuestra obligación procurar que esta influencia sea para beneficio de éste. Además, debemos informar al paciente de esta cualidad de la terapia. c) Finalmente, una situación distinta es la forma de cuantificar -o "numerizar"esta posibilidad de cambio. No debemos perder la perspectiva que los diferentes valores utilizados se han generado en poblaciones distintas a la nuestra, y que los parámetros para juzgar la estética varían de lugar en lugar y de cultura a cultura (2).

El propósito del presente estudio fue comparar los resultados a nivel del perfil blando de pacientes tratados ortodónticamente con y sin extracciones de primeros premolares.

\section{MATERIAL Y MÉTODOS}

Se emplearon 48 radiografías cefalométricas pre y post operatorias correspondientes a 24 pacientes. De estos, 12 fueron tratados con extracciones de las cuatro primeras premolares (E); y 12 fueron tratados sin extracciones, estos pacientes constituyeron el grupo control (NE). Todos eran portadores de 
maloclusión de Clase I de Angle, los criterios para decidir las extracciones fueron: nivel la discrepancia alveolo dentaria y el nivel de inclinación de las pieza anteriores.

La toma radiográfica antes y después del tratamiento fue realizada en las mismas condiciones, con los pacientes ubicados con el plano de Frankfort paralelo al piso a $1,52 \mathrm{~cm}$ de distancia. En todos los casos se empleó la técnica estándar Edgewise.

Para la evaluación se utilizaron medidas angulares y lineales: 6 medidas para evaluar ántero posteriormente el labio superior, 6 para la evaluación del labio inferior también en sentido ántero posterior,

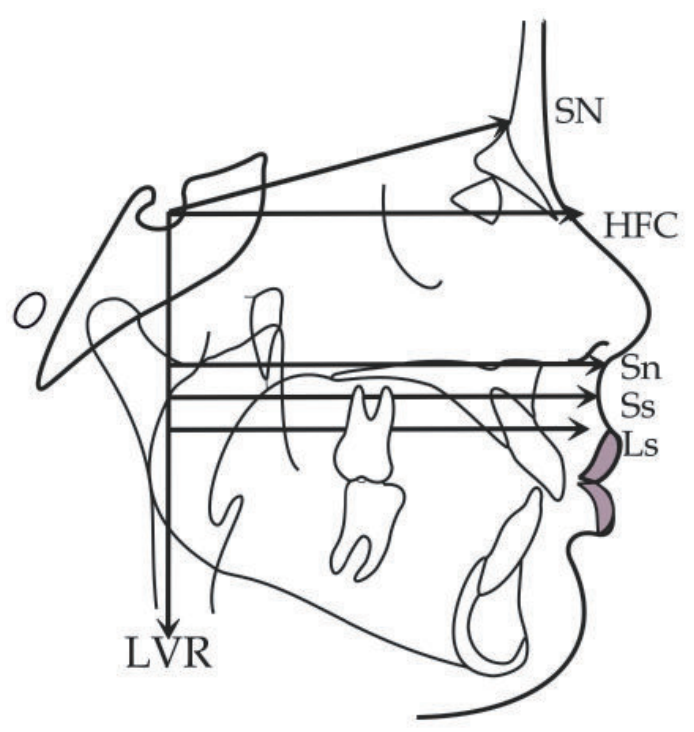

Figura 1. Referencia posterior, media y anterior para evaluación del labio superior.
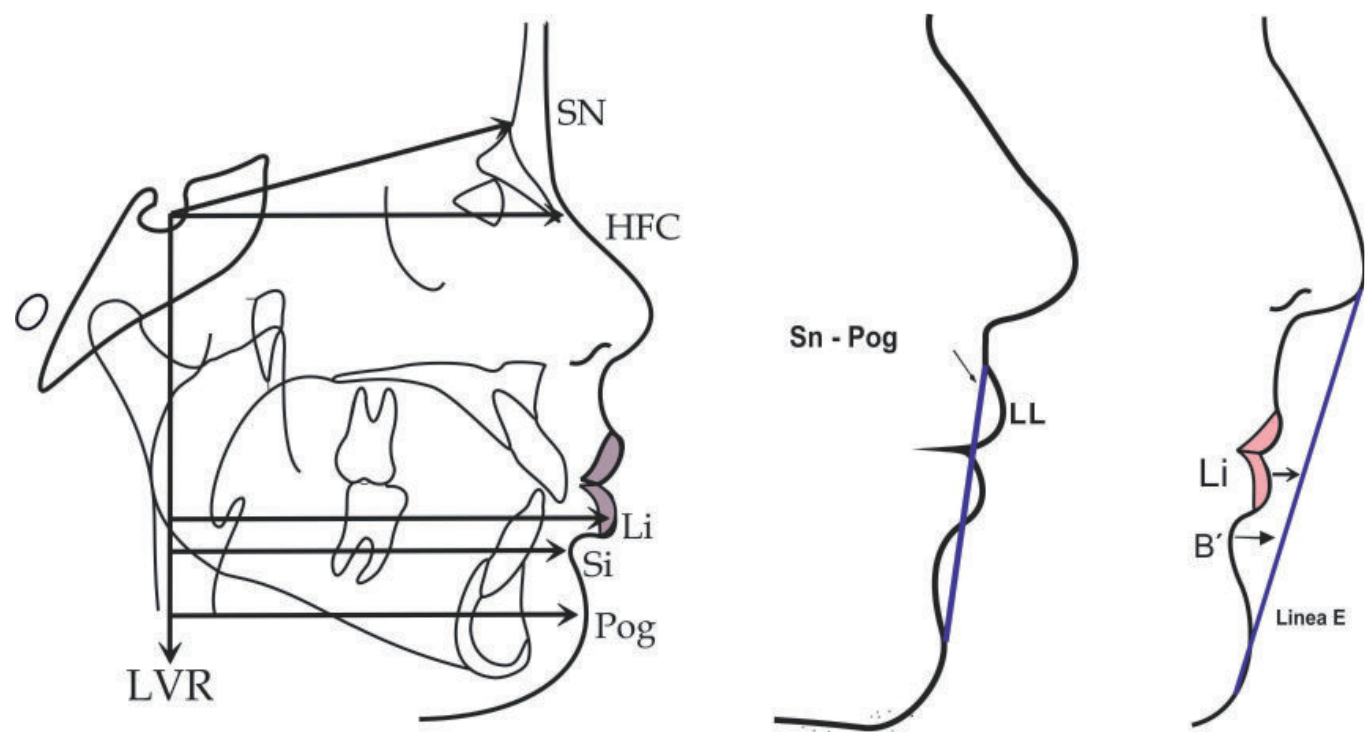

Figura 2. Referencia posterior, media y anterior para la evaluación del labio inferior. 
El labio inferior, también en el sentido ántero posterior, fue evaluado tomando como referencia las 3 líneas antes mencionadas (Figura 2).

Además, en la evaluación de ambos labios se empleó el ángulo nasolabial para el labio superior, labiomental para el inferior y el ángulo $\mathrm{Z}$ para el perfil total.

La evaluación vertical se realizó mediante el análisis de la altura del tercio inferior de la cara, representado por la distancia SN-Me; éste se consideró dividido en dos partes: una parte superior, representada por la distancia Sn-St y otra inferior, representada por la distancia St-Me, conforme lo indican Dobrocky y Smith (2).

Para el análisis comparativo, utilizamos la prueba t de Student para muestras pareadas o antes y después (en este caso, pre operatoria y post operatoriamente). El nivel de significancia aceptado fue de 95\%.

\section{RESULTADOS}

Las características de ambos grupos se pueden apreciar en la tabla 1.

En el grupo tratado sin extracciones, al realizar el análisis mediante la prueba t de Student para muestras pareadas, entre las mediciones efectuadas antes y después del tratamiento encontramos variaciones significativas sólo en la posición del labio superior, indicando una protrusión del mismo, expresada por el aumento de las distancias SN-LVR y Ss-LVR, cuando la medición se realizó empleando la referencia posterior; cuando empleamos la referencia anterior, nos indicó una retrusión del mismo expresada por el aumento de la distancia A'-E. Verticalmente, hallamos un aumento en la altura del tercio inferior de la cara, fundamentalmente en la parte inferior de dicho tercio, expresado por el aumento de la distancia St-Me, trabajando a un nivel de confianza de 0,05. Estos resultados son presentados en la tabla 2.

En el grupo tratado con extracciones, al realizar el análisis mediante la prueba $t$ de Student para muestras pareadas, entre las mediciones efectuadas antes y después del tratamiento encontramos variaciones significativas en 14 de los 18 indicadores estudiados, trabajando con un nivel de confianza de 0,05. El labio superior analizado con la referencia posterior mostró una protrusión, mientras que cuando se analizó con las referencias media y anterior se comportó retrusivamente. El labio inferior tuvo el mismo comportamiento. Verticalmente, hubo un aumento del tercio inferior de la cara. Estos cambios son resumidos en la tabla 3 .

Tabla 1. Características de los grupos de estudio.

\begin{tabular}{lccc}
\hline Grupo & Sexo & Edades (promedio) & Tiempo de Tx (promedio) \\
\hline $\mathrm{E}$ & $6 \mathrm{H}, 6 \mathrm{M}$ & $12,5(\mathrm{a})$ & 26 meses \\
$\mathrm{NE}$ & $8 \mathrm{H}, 4 \mathrm{M}$ & $13(\mathrm{a})$ & 24 meses \\
$\mathrm{E}=$ exodoncia & & \\
$\mathrm{NE}=$ no exodoncia & & \\
\hline
\end{tabular}

Tabla 2. Indicadores con cambios significativos en el grupo sin extracciones.

\begin{tabular}{lcccc}
\hline \multicolumn{1}{c}{ Indicador } & $\begin{array}{c}\text { Razón t } \\
\text { calc. }\end{array}$ & Razón t tabla & Grados de lib. & Signif. 2 colas \\
\hline Dist. Sn-LVR & $-2,717$ & 2,201 & 11 & Significativa \\
Dist. Ss-LVR & $-2,4$ & 2,201 & 11 & Significativa \\
Dist. A'-E & 2,803 & 2,201 & 11 & Significativa \\
Dist. St-Me & $-3,156$ & 2,201 & 11 & Significativa \\
\hline
\end{tabular}


Tabla 3. Indicadores con cambios significativos en el grupo con extracciones.

\begin{tabular}{lcccl}
\hline Indicador & Razón t cal. & Razón t tabla & Grad. de lib. & Signif. 2 colas \\
\hline Ángulo Z & $-4,501$ & 2,201 & 11 & Significativa \\
Distancia Sn-LVR & $-2,715$ & 2,201 & 11 & Significativa \\
Distancia Ss-LVR & $-2,399$ & 2,201 & 11 & Significativa \\
Distancia Ls-LVR & $-2,335$ & 2,201 & 11 & Significativa \\
Distancia Ul-SnPog & 2,982 & 2,201 & 11 & Significativa \\
Distancia UL-E & 5,17 & 2,201 & 11 & Significativa \\
Distancia A'-E & 4,799 & 2,201 & 11 & Significativa \\
Distancia Si-LVR & $-3,606$ & 2,201 & 11 & Significativa \\
Distancia Pog-LVR & $-4,607$ & 2,201 & 11 & Significativa \\
Distancia Ll-SnPog & 4,368 & 2,201 & 11 & Significativa \\
Distancia Ll-E & 2,857 & 2,201 & 11 & Significativa \\
Distancia B'-E & 4,367 & 2,201 & 11 & Significativa \\
Distancia Sn-Me & $-2,675$ & 2,201 & 11 & Significativa \\
Distancia St-Me & $-3,82$ & 2,201 & 11 & Significativa \\
\hline
\end{tabular}

Tabla 4. Cambios observados entre ambos grupos.

\begin{tabular}{lcccc}
\hline \multicolumn{1}{c}{ Indicadores } & Razón t calc. & $\begin{array}{c}\text { Razón t } \\
\text { tabla }\end{array}$ & $\begin{array}{c}\text { Grad. } \\
\text { de lib. }\end{array}$ & $\begin{array}{c}\text { Signif. 2 } \\
\text { colas }\end{array}$ \\
\hline Ángulo Z & $-1,104$ & 2,201 & 11 & N.E. \\
Ángulo nasolabial & 0,74 & 2,201 & 11 & N.E. \\
Ángulo labiomental & 0,342 & 2,201 & 11 & N.E. \\
Distancia Sn-LVR & $-0,065$ & 2,201 & 11 & N.E. \\
Distancia Ss-LVR & $-0,109$ & 2,201 & 11 & N.E. \\
Distancia Ls-LVR & $-0,387$ & 2,201 & 11 & N.E. \\
Distancia Ul-SnPog & 0,137 & 2,201 & 11 & N.E. \\
Distancia Ul-E & $-0,133$ & 2,201 & 11 & N.E. \\
Distancia A'-E & 0,488 & 2,201 & 11 & N.E. \\
Distancia Ll-LVR & $-0,17$ & 2,201 & 11 & N.E. \\
Distancia Si-LVR & $-1,213$ & 2,201 & 11 & N.E. \\
Distancia Pog-LVR & $-0,873$ & 2,201 & 11 & N.E. \\
Distancia Ll-SnPog & 0,869 & 2,201 & 11 & N.E. \\
Distancia Ll-E & 0,782 & 2,201 & 11 & N.E. \\
Distancia B'-E & $-0,421$ & 2,201 & 11 & N.E. \\
Distancia Sn-Me & $-0,848$ & 2,201 & 11 & N.E. \\
Distancia Sn-St & $-0,738$ & 2,201 & 11 & N.E. \\
Distancia St-Me & $-0,649$ & 2,201 & 11 & N.E. \\
\hline
\end{tabular}


Al comparar los grupos con y sin extracciones encontramos que ninguno de los 18 indicadores estudiados presentó cambios estadísticamente significativos; la razón t obtenida fue siempre menor que la de la tabla $(t=2,2010)$, trabajando con 11 grados de libertad y al 0,05 de confianza. Los resultados son presentados en la tabla 4.

\section{DISCUSIÓN}

Haber obtenido resultados que estadísticamente no muestren valores de significancia en ninguna de las 18 variables estudiadas, nos llevaría a pensar que los tratamientos con extracciones o sin ellas, en términos del perfil, resultan similares. Sin embargo, hay que considerar que las extracciones no implican necesariamente una mecánica retrusiva y que no son el único factor a considerar como generador de modificaciones del perfil.

Barrer y Ghafari (8) realizaron un trabajo en el que compararon siluetas de perfiles de 48 pacientes portadores de maloclusiones Clase II división 1 con retronagtismo mandibular, antes y después del tratamiento ortodóncico realizado sin extracciones, tratados de cuatro diferentes formas, y evaluados por estudiantes de odontología. Ellos concluyeron que los perfiles post tratamiento fueron considerados como mejores que los del pretratamiento.

Bishara y Jakobsen (9) también evaluaron siluetas de perfiles de pacientes de tres diferentes grupos con oclusión normal, con tratamiento con extracciones, y con tratamiento sin extracciones; llegando a la conclusión que los individuos en general observan: a) que las personas con oclusión normal tienen un perfil más agradable que aquellos que presentan maloclusiones, b) que el tratamiento ortodóncico genera cambios en la apariencia facial, c) que estos cambios son percibidos como favorables, tanto en aquellos pacientes en los que se realizaron extracciones, como en los que no se les realizó, d) que inmediatamente después del tratamiento, estos cambios se percibieron como más favorables en el grupo al que se les realizó extracciones, e) que luego de dos años de finalizada la terapia, no fue posible percibir una diferencia entre los perfiles de los tres grupos, f) que el cambio se percibió como mejor en pacientes del sexo femenino.
Este tipo de estudios toman distancia de los que procuran sostener, mediante valores numéricos solamente, la influencia del tratamiento sobre el perfil y van aún más allá, puesto que evalúan la percepción de las personas para con la terapia: a los pacientes les interesa mejorar, más que estar dentro de los rangos "normales" de tal o cual medición.

Bishara y col. (10) señalan que si bien el crecimiento en la niñez y la adolescencia ha sido bien documentado, durante la adultez ocurren cambios que deben ser tomados en cuenta en el momento de plantear determinada mecánica de tratamiento. Ellos evaluaron 30 pacientes normales, 15 hombres y 15 mujeres entre los 25 y 46 años, y encontraron que en ambos casos los labios superior e inferior se ubicaron más retrusivos que la nariz y el mentón con el paso de los años. La implicancia clínica de este hallazgo nos indica que debemos evitar finalizar los casos con perfiles muy rectos o retrusivos, ya que como consecuencia de la edad, este tipo de características se van a exagerar.

Hershey (11) sostiene, que los investigadores son aún incapaces de aislar los efectos del tratamiento en los cambios del perfil debido a la super imposición del crecimiento de las estructuras en evaluación y de las estructuras óseas subyacentes, esto permite comprender el resultado de no hallar diferencias entre nuestros grupos de estudio.

Así pues, el presente hallazgo concuerda con los resultados obtenidos por la mayoría de investigadores $(2,7,8,9)$. Sin embargo, existen otros autores $(3,4)$ que señalan diferencias significativas entre los perfiles pre y postoperatorios de pacientes tratados con extracciones.

Caplan y Shivapuya (3) encontraron variación tanto en los tejidos del perfil blando como en los tejidos dentarios, en una muestra de 28 adultos de sexo femenino y raza negra, tratados con extracciones de las cuatro primeras premolares. Respecto a este estudio, debemos considerar que es uno de los casos donde la posibilidad de generar cambios está más marcada: la biprotrusión dentaria, propia de la raza negra, conlleva a un perfil bastante más lleno que el aceptado como ideal; de allí que desborde las normas de índole clínico y cefalométrico. Además, es conveniente puntualizar que en este caso no se 
estableció ningún grupo control. Asimismo, cabe resaltar que pese a los valores de significancia hallados, el mencionado estudio sostiene que es muy difícil predecir el comportamiento del labio superior cuando se retrae el sector dentario anterior; intuitivamente supondríamos que los labios van a seguir al tejido duro (modificando con esto el perfil), pero la variación individual es sumamente alta. Desde otra óptica debemos apreciar el comportamiento del labio inferior, que sí exhibe una alta correlación con la retracción del sector ántero inferior, resultados que concuerdan con los obtenidos por Burstone (12).

Una referencia frecuentemente utilizada para determinar cambios a nivel de los labios es el ángulo nasolabial. En nuestro estudio esta medida no mostró cambios estadísticamente significativos, ni en el grupo tratado con extracciones ni en el que fue tratado sin ellas, ni en la comparación entre ambos grupos.

Robinson (13) realizó un estudio en pacientes edéntulos, alterando la posición de los incisivos, de manera que tuvieran angulaciones distintas en las prótesis de cada uno. Pudo observar que el ángulo nasolabial variaba según la posición de los incisivos: el patrón que advirtió fue que si los incisivos se colocaban más hacia lingual, el labio superior seguía esa posición; en contraparte, si se ubicaban más hacia vestibular, el labio se protruía. Si trasladamos estas conclusiones a los casos donde realizamos extracciones, la posición final de los labios debería ser más retrusiva que la que existía antes de la terapia, obteniéndose un ángulo más obtuso; en los casos donde no se realizaran extracciones, la posibilidad iría en el sentido de protruir el labio en la medida que las piezas dentarias se vestibulizarían por la mecánica, obteniéndose un ángulo nasolabial más agudo. Estas aproximaciones tienen mucho de sentido común, tal como lo reportan Drobocky y Smith (2) quienes encontraron en su estudio un aumento en promedio de 5.2 grados en el ángulo nasolabial luego del tratamiento ortodóntico, pero no dejan de resaltar la variabilidad individual de los resultados, indicando que el $26,3 \%$ de los sujetos estudiados terminaron con un ángulo nasolabial más agudo que aquel con el que comenzaron el tratamiento. Igualmente, Waldman (14) al analizar 41 casos tratados con extracciones, pudo encontrar que 17 de ellos no sólo no aumentaron el valor del ángulo nasolabial sino que lo disminuyeron luego de la terapia, presentando un ángulo más agudo al final del tratamiento. Nuevamente es posible apreciar cómo las respuestas individuales son las que resaltan en los diferentes estudios.

Cabe mencionar que, en el estudio donde se analizan a pacientes edéntulos, debemos tomar en cuenta que son pacientes cuyos tejidos blandos se han enfrentado durante mucho tiempo a la ausencia de un soporte dentario, con el consiguiente cambio en la tonicidad muscular que afecta las dimensiones y posiciones de estos tejidos; en cuanto a las referencias posteriores, donde se trabajó con pacientes a los que recién se les había terminado sus respectivos tratamientos, es posible considerar un periodo de ajuste a nivel de tejidos blandos, a fin de que estos puedan conseguir su posición y dimensiones finales.

La literatura especializada no tiene elementos para poder afirmar que la realización de extracciones o no, tenga como resultado determinado patrón específico de perfil $(15,16)$. La información disponible nos brinda orientaciones y pautas generales, las decisiones clínicas deben basarse en evaluar al paciente integralmente para escoger la terapéutica más adecuada.

\section{CONCLUSIONES}

En función de los resultados obtenidos podemos concluir que:

- No se hallaron diferencias estadísticamente significativas entre los valores que evalúan los cambios ántero posteriores y verticales en el grupo estudiado de pacientes tratados ortodóncicamente con extracciones y sin ellas.

- En el grupo tratado con extracciones, tanto el labio superior como el inferior, mostraron un aumento estadísticamente significativo en las medidas que evaluaron su posición ántero posterior. Tal situación debe interpretarse como que los labios no sólo no se retruyeron, sino que pese a las extracciones se colocaron más protrusivos. Esta afirmación es válida cuando se hace en función de la referencia posterior (LVR).

- El labio superior evaluado ántero posteriormente en el grupo tratado sin extracciones, presentó un aumento estadísticamente significativo sólo en dos de las medidas que analizan los cambios en este plano; ambas distancias medidas en función a la referencia posterior. Adicionalmente, una 
medida analizada en función de la referencia anterior mostró un aumento estadísticamente significativo, lo cual indica una retrusión labial en función de la línea $\mathrm{E}$.

- El labio inferior en el grupo tratado sin extracciones, no mostró cambios estadísticamente significativos en la evaluación ántero posterior.

- Los valores cefalométricos que evalúan los cambios verticales, mostraron en ambos grupos incrementos estadísticamente significativos en sus valores, fundamentalmente en la mitad inferior del tercio inferior de la cara.

\section{Correspondencia:}

Herbert Orrego Carrillo

Av. Las Palmeras 190 - Dpto 201, San Isidro. Lima, Perú.

Correo electrónico: herbertorrego@hotmail.com

\section{REFERENCIAS BIBLIOGRÁFICAS}

1. Looi LK, Mills JR. The effect of two contrasting forms of orthodontics treatment on the facial profile. Am J Orthod. 1986; 89(6):507-517.

2. Drobocky OB, Smith RJ. Changes in facial profile during orthodontic treatment with extraction of four first premolars. Am J Orthod Dentofacial Orthop. 1989; 95(3):220-30.

3. Caplan M, Shivapuja P. The effect of premolar extractions on the soft profile in adult African American females. Angle Orthodontist. 1997; 67(2):129-136.

4. Witzig J, Yerkes I. Funcional jaw orthopedics: mastering more than technique. En: Gelb $\mathrm{H}$ (ed): Clinical management of head, neck and TMJ pain and dysfunction. $2^{\text {a }}$ ed. Philadelphia, E.E.U.U.: W Sanders; Co; 1985.

5. James RD. A comparative study of facial profiles in extraction and nonextraction treatment. Am J Orthod Dentofacial Orthop. 1998; 114(3):265-76.
6. Paquette DE, Beattie JR, Johnston LE Jr. A long-term comparision of nonextraction and premolar extraction edgewise therapy in "borderline" Class II patients. Am J Orthod Dentofacial Orthop. 1992; 102(1):1-14.

7. Rains MR, Nanda R. Soft tissue changes associated with maxillary incisor retraction. Am J Orthod. 1982; 81(6):481-8.

8. Barrer JG, Ghafari J. Silhouette profiles in the assessment of facial esthetics: a comparison of cases treated with various orthodontic appliances. Am J Orthod. 1985; 87(5):385-91.

9. Bishara SE, Jakobsen JR. Profiles changes in patients treated with and without extractions: assessments by lay people. Am J Orthod Dentofacial Orthop. 1997; 112(6):639-44.

10. Bishara SE, Treder JE, Jakobsen JR Facial and dental changes in adulthood. Am J Orthod Dentofacial Orthop. 1994; 106(2):175-86.

11. Hershey HG. Incisor tooth retraction and subsequent profile change in post adolescent female patients. Am J Orthod. 1972; 61(1):45-54.

12.Burstone CJ. The integumental contour and extension patterns. Angle Orthod.1959;29(2):93-104.

13. Robinson R. Changes in lip position in coincidence with movement of artificial teeth [Tesis de Maestría]. Indiana, USA: IndianaUniversity, 1960.

14.Waldman B. Change in lip contour with maxillary incisor retraction. Angle Orthod.1982; 52(2):129-134.

15. Wholley C. The effects of commonly prescribed premolar extraction sequence on the curvature of the upper and lower lips. Angle Orthod. 2003; 73(4):38695.

16.Aslihan E. Profile changes of patients treated with and without premolar extractions Am J Orthod Dentofacial Orthop. 2007; 132(3):324-31.

Recibido: 21/06/2014 Aceptado: 01/08/2014 